\title{
The Potential Anti-Diabetic Effects of Saskatoon Berry in Experimental Mouse Models
}

\author{
Moghadasian $\mathrm{MH}^{1,2,5 *}$, Masisi $\mathrm{K}^{1,2}$, Le K ${ }^{2}$, Beta $\mathrm{T}^{1}$, \\ Shen $\mathbf{G X}^{2,3}$ and Fischer $\mathbf{G}^{4}$ \\ ${ }^{1}$ Department of Food \& Human Nutritional Sciences, \\ Canada \\ ${ }^{2}$ Canadian Centre for Agri-food Research in Health and \\ Medicine, University of Manitoba, Canada \\ ${ }^{3}$ Departments of Physiology and Internal Medicine, \\ Canada \\ ${ }^{4}$ Department of Pathology, University of Manitoba, \\ Canada \\ ${ }^{5}$ International Nutrition Research Inc, Winnipeg, Canada \\ *Corresponding author: Moghadasian MH, Canadian \\ Centre for Agri-food Research in Health and Medicine, \\ Winnipeg, MB, Canada
}

Received: December 05, 2018; Accepted: J anuary 08, 2019; Published: January 15, 2019

\begin{abstract}
We previously reported that Saskatoon-Berry-Powder (SBP) might have beneficial effects on vascular inflammation and endoplasmic reticulum stress in experimental animals. In a pilot study, we tested various oral doses of SBP and learned that the $5 \%(\mathrm{w} / \mathrm{w})$ dose seems to have the best effects. This study investigated the impact of dietary intake of SBP at $5 \%(\mathrm{w} / \mathrm{w})$ on several biochemical factors pertaining to body weight gain, glucose metabolism; lipids, inflammation and organ function over a 4-week study period. The $\mathrm{db} / \mathrm{db}$ mouse model of type 2 diabetes and their wild-type counterparts (C57BL/6J mice) were used. Body weight, food-intake, whole blood glucose, and plasma total cholesterol levels were significantly higher in the $\mathrm{db} / \mathrm{db}$ mice as compared with those in C57BL/6J mice. Consumption of SBP did not change the animals body weight gain rates or food consumption in either animal model over the experimental course. The SBP-treated db/db mice showed approximately 30\% reductions in their blood and urine glucose levels as compared to those in nontreated $\mathrm{db} / \mathrm{db}$ mice over 4 weeks of experiment. Furthermore, the $\mathrm{db} / \mathrm{db}$ mice show a different inflammatory-marker profile as compared to that in C57BL/6J mice; treatment with SBP was associated with changes in such profiles mainly in $\mathrm{db} / \mathrm{db}$ mice. It is not clear which component(s) of SBP is responsible for the effects observed in this study. Future studies warrant identification of such functional components of SBP.
\end{abstract}

Keywords: Saskatoon berry powder; C57BL/6J mice; db/db mice; Inflammatory marker; Plasma lipids

\section{Introduction}

Current dietary guidelines from several countries recommend regular consumption of fruits and vegetables to improve quality of life. Fruits and vegetables are nutrient-dense foods with significant amounts of phytochemicals. Phytochemicals are chemical compounds naturally available in plant-derived foods. These health-promoting substances belong to a number of chemical families.

Berries are generally implied to "small fruits" and include blueberries, raspberries, blackberries, Saskatoon berries, cranberries and others. All of these fruits are known for their high concentrations of antioxidant phytochemicals [1]. reported that high tocol contents of seed oil from blueberries, black raspberries and blackberries are responsible for the antioxidant properties of the seed oils. Another recent study reported significant antioxidant and anti-inflammatory properties of methanolic extracts of Goji berry, blueberry and cranberry in both in vitro and in vivo settings [2]. Similarly, blueberry anthocyanins reduced the oxidative stress status in endothelial cells [3]. Furthermore, orally-administered blueberry extract prevented the development of Dextran Sodium Sulfate (DSS)-induced ulcerative colitis in mice [4]. Anti-inflammatory and antioxidant activities of blueberry derived pterostilbene were reported to be responsible for prevention of corneal epithelial cell injuries in an in vitro model [5]. Consumption of blueberries at $8 \%(\mathrm{w} / \mathrm{w})$ over 16 weeks was associated with prevention of Diethyl Nitrosamine (DEN)-induced hepatic cirrhosis and pre-neoplastic lesions in rats; this effect was thought to be mediated through antioxidant properties of blueberries [6].

Purified ellagitannins from strawberries had antihypertensive and hypoglycemic activities in an in vitro experimental setting [7]. Extracts from six common berries, including blackberry, black raspberry, blueberry, cranberry, red raspberry and strawberry were rich in phenolic compounds such as anthocyanins, flavonols, flavanols, ellagitannins, gallotannins, proanthocyanidins, and phenolic acids. These extracts revealed anti-proliferative activities in an in vitro model using human oral, breast, colon and prostate cancer cell lines. Furthermore, these extracts showed pro-apoptosis activities in a colon cell line [8].

Saskatoon Berries (SB) grow naturally or are planted in the Canadian prairie area and northern states of the United States of America. Saskatoon berry powder has previously shown to be relatively higher in anthocyanins compared to blue berries, strawberries, raspberries, chockecherry or seabuckthorn [9]. However, the effects of Saskatoon Berry Powder (SBP) on metabolic risk factor have not been well documented. Therefore, we hypothesis that daily consumption of SBP reduces metabolic risk factors in an animal model of diabetes. To test our hypothesis, the present study investigated the impact of daily consumption of SBP on metabolic risk factors in an animal model of diabetes, namely $\mathrm{db} / \mathrm{db}$ mice and their wild-type counterparts-C57BL/6J mice. 


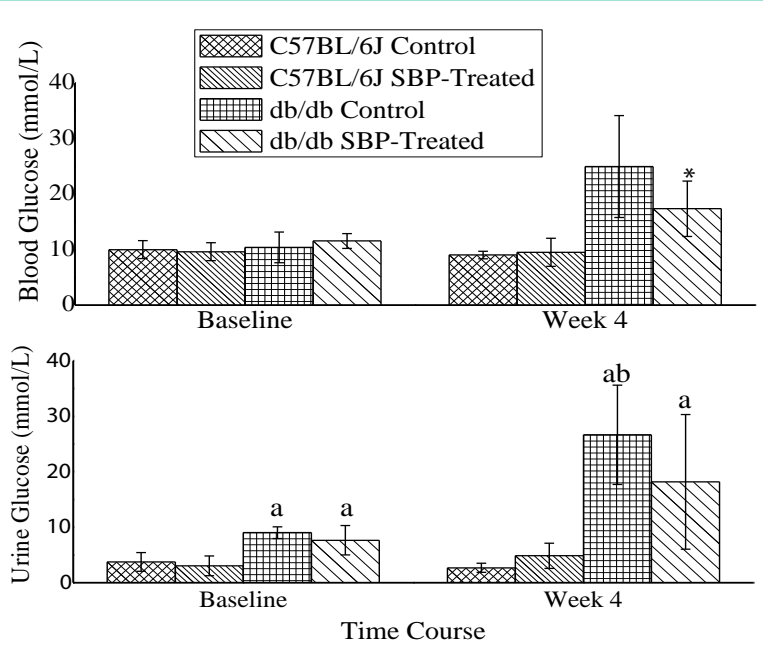

Figure 1: Upper Panel: Effects of Saskatoon Berry Powder (SBP) on whole blood glucose levels in both $\mathrm{db} / \mathrm{db}$ mice and their wild-type counterparts. ${ }^{*} \mathrm{P}<0.05$ SBP-treated mice as compared to SBP-treated C57BL/6J mice. Lower Panel: Effects of Saskatoon Berry Powder (SBP) treatment on urine glucose levels in both $\mathrm{db} / \mathrm{db}$ mice and their wild-type counterparts.

Means $\pm \mathrm{SD} ; \mathrm{n}=8$.

a, $P<0.05$ as compared to corresponding $\mathrm{C} 57 \mathrm{BL} / 6 \mathrm{~J}$ groups at baseline b, $\mathrm{P}<0.05$ as compared to either of $\mathrm{C} 57 \mathrm{BL} / 6 \mathrm{~J}$ group at week 4

\section{Materials and Methods}

\section{Saskatoon berry powder}

Fresh Smoky Saskatoon Berry was purchased from Prairie Lane Saskatoon (Portage La Prairie, Manitoba). The berries were cleaned and frozen at $-80^{\circ} \mathrm{C}$ and then lyophilized using a freeze-drier without an addition of supplement. Dried whole berries were powered and stored in vacuum-sealed bags at $-20^{\circ} \mathrm{C}$. All processes were conducted in sterile stainless steel containers.

\section{Animals}

Male $\mathrm{db} / \mathrm{db}$ and their C57BL/6J wild-type counterpart mice (32 mice of 6 weeks of old) were purchased from the Jackson Laboratory (Bar Harbor, ME, USA). During one-week quarantine period in the animal facility at St. Boniface Hospital Albrechtsen Research Centre (Winnipeg, MB, Canada), the mice were fed with standard mouse chow. The mice were housed in a temperature-controlled room at $22^{\circ} \mathrm{C}-24^{\circ} \mathrm{C}$, with a $12: 12$-h light-dark cycle. After one week, animals were randomized into indicated groups ( $\mathrm{n}=8 /$ group). Wildtype mice and $\mathrm{db} / \mathrm{db}$ mice received regular chow or the same chow supplemented with $5 \%(\mathrm{w} / \mathrm{w})$ SBP for 4 weeks as optimized in our previous study. All experimental diets were prepared in the form of pellets. Body weights and food consumption were measured weekly. The experimental period was 4 weeks. During the experiments, the animals were housed in pairs using conventional mouse cages. All animals had ad libitum access to food and water. The foods were provided to animals on a weekly schedule. The leftover food was discarded. The food was made as needed, and stored in dark bags, and closed containers in a cold room $\left(\right.$ at $\left.4^{\circ} \mathrm{C}\right)$ until used. At the end of the experiment, final blood samples were taken from the heart; plasma samples were prepared and pooled for further biochemical analyses. The protocol for animal experiments was approved by the Animal Protocol Management and Review Committee at the University of
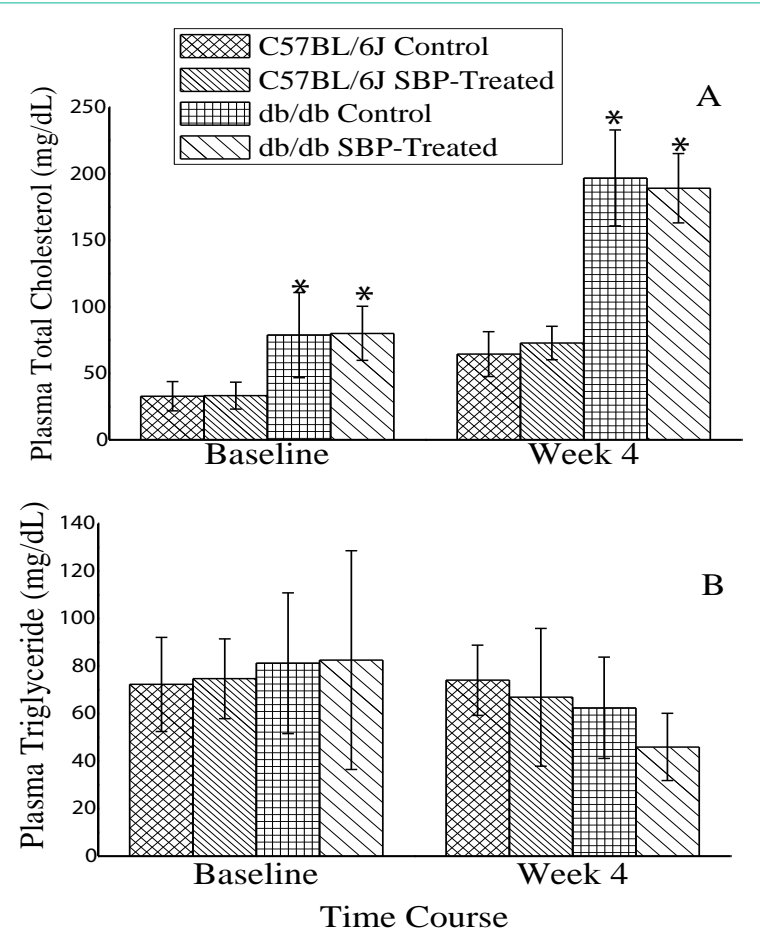

Figure 2: Effects of SBP on plasma cholesterol and triglycerides levels in both $\mathrm{db} / \mathrm{db}$ mice and their wild-type counterparts. ${ }^{*} \mathrm{P}<0.05 \mathrm{db} / \mathrm{db}$ mice as compared to C57BL/6J mice. Means \pm SD; $n=8$.

Manitoba. The mice were maintained according to the Guide and Care and Use of Laboratory Animals approved by University of Manitoba Animal Care Committee (Winnipeg, MB, Canada).

\section{Blood Sampling and Biochemical Analyses Blood sampling}

All mice were fasted for approximately 4 hours prior to blood collection. Blood samples were drawn through jugular vein of lightly anesthetized animals during the study and through cardiac puncture at the end of the study. The whole blood samples were then centrifuged at $3000 \mathrm{rpm}$ for $10 \mathrm{~min}$ at $4^{\circ} \mathrm{C}$ to yield the plasma fraction, which was used for subsequent analyses.

\section{Whole blood glucose levels}

The levels of glucose in mouse blood samples were measured using Alpha TRAK glucose monitoring system from Abbott. This measurement was performed on the samples taken weekly from the jugular vein during the study course and final blood sample taken at the end of the study.

\section{Plasma lipid levels}

Plasma levels of Total Cholesterol (TC) and Triglyceride (TG) were estimated using our standard enzymatic procedures [10]. These measurements were performed on plasma samples prepared from the final blood collection.

\section{Plasma cytokine profile}

Plasma samples from final blood collection were pooled. Four pooled samples from each experimental group were sent to Ray Biotech Inc. (Norcross, GA, USA) for estimation of concentrations of 
Table 1: Body weight and food intake among various groups of mice over the experimental course.

\begin{tabular}{|c|c|c|c|c|c|}
\hline \multirow{2}{*}{ Measurements } & Time point & \multicolumn{2}{|c|}{ db/db mice } & \multicolumn{2}{|c|}{ C57BL/6J mice } \\
\hline & & Control $(n=8)$ & SBP-treated $(n=8)$ & Control $(n=8)$ \\
\hline Body weight $(\mathrm{g})$ & Baseline & $23.9 \pm 3.5^{\mathrm{a}}$ & $26.8 \pm 1.9^{\mathrm{a}}$ & $17.6 \pm 2.3$ \\
\hline & Week 4 & $45.7 \pm 3.7^{\mathrm{a}}$ & $46.0 \pm 1.7^{\mathrm{a}}$ & $20.9 \pm 2.6$ \\
\hline Food Intake $(\mathrm{g}) /$ day & Baseline & $14.3 \pm 1.9^{\mathrm{a}}$ & $14.1 \pm 1.9^{\mathrm{a}}$ & $4.6 \pm 0.3$ \\
\hline & Week 4 & $11.3 \pm 1.3^{\mathrm{a}}$ & $10.3 \pm 0.5^{\mathrm{a}}$ & $4.7 \pm 1.0$ \\
\hline
\end{tabular}

SBP: Saskatoon Berry powder

a, $\mathrm{P}<0.05$ as compared to corresponding $\mathrm{C} 57 \mathrm{BL} / 6 \mathrm{~J}$ mice

Values are means $\pm \mathrm{SD}$

21 common inflammatory markers.

\section{Statistical analysis}

Eight mice were used in each group in order to have sufficient statistical power to detect statistically significant differences at $\mathrm{P}<0.05$ and a power of $80 \%$ in values from biological samples. Data were analyzed using one-way ANOVA followed by the application of the Tukey test to determine the statistically significant differences among the groups. All results are presented as means \pm Standard Deviation (SD).

\section{Results}

\section{Body weight and food intake}

Table 1 summarizes food intake and body weight gain during the study course. All mice gained weight comparably regardless of the treatment protocol. Body weight and food intake were significantly $(\mathrm{P}<0.05)$ higher in the $\mathrm{db} / \mathrm{db}$ diabetic mice as compared with those in $\mathrm{C} 57 \mathrm{BL} / 6 \mathrm{~J}$ mice. Baseline mean body weight was approximately $25 \%$ higher in $\mathrm{db} / \mathrm{db}$ mice than that in $\mathrm{C} 57 \mathrm{BL} / 6 \mathrm{~J}$ mice (25.3 grams $v$ s 18.5 grams, $\mathrm{n}=16$ for each group). The amounts of the average weight gain over the 4 weeks of experimental course were 20.5 grams and 3.2 grams in $\mathrm{db} / \mathrm{db}$ and C57BL/6J mice, respectively. However, estimated food intake was not significantly increased over the experimental course in either of the animal model. Consumption of SBP did not change the animals' body weight gain rates or food consumption in either animal model over the experimental course.

\section{Blood and urine glucose levels}

The blood glucose levels are shown on upper Panel of (Figure 1). At baseline, all of the animals had comparable whole blood glucose levels. However, by the end of the study both groups of $\mathrm{db} / \mathrm{db}$ mice had significantly higher blood glucose levels as compared to those in either group of wild type mice. On the other hand, the $\mathrm{db} / \mathrm{db}$ mice treated with SBP had approximately $30 \%$ lower blood glucose levels as compared to those in non-treated $\mathrm{db} / \mathrm{db}$ control mice. Such glucoselowering effects of SBP were not seen in wild-type mice.

The levels of glucose in urine samples from $\mathrm{db} / \mathrm{db}$ mice were statistically higher at baseline as compared with those in wild-type mice. By the end of the study, both groups of $\mathrm{db} / \mathrm{db}$ mice had much higher amounts of glucose in their urine samples as compared to the baseline data or those in the wild-type mice at the end of the study. It is interesting that consumption of SBP in $\mathrm{db} / \mathrm{db}$ mice resulted in $30 \%$ reductions in urine glucose levels, while a non-significant increase of $10 \%$ was noticed in the urine of wild-type mice. Urine glucose levels are shown on the lower Panel of (Figure 1).

\section{Plasma lipid levels}

The plasma Total Cholesterol (TC) and Triglyceride (TG) levels at the beginning and end of the study are shown on (Figure 2). As shown in Panel A, plasma TC levels were significantly higher in $\mathrm{db} / \mathrm{db}$ mice as compared to those in C57BL/6J mice at baseline. However, by the end of the study the $\mathrm{db} / \mathrm{db}$ mice developed hypercholesterolemia. Treatment with SBP was not able to alleviate the degree of hypercholesterolemia in $\mathrm{db} / \mathrm{db}$ mice. Both groups of $\mathrm{db} / \mathrm{db}$ mice had significantly higher TC levels as compared with those in C57BL/6J mice at week 4. Panel B of (Figure 2) demonstrates comparable concentrations of TG in all four groups of mice at baseline. However, unlike TC levels, the levels of TG did not increase by week $4 \mathrm{in} \mathrm{db} /$ $\mathrm{db}$ mice. Furthermore, by the end of the study, SBP treatment was associated with a non-significant reduction in TG levels in $\mathrm{db} / \mathrm{db}$ mice only.

\section{Plasma inflammatory markers}

Table 2 summarizes various common plasma inflammatory markers detected in pool plasma samples of all groups of experimental mice. A comparison between the control wild-type mice and control $\mathrm{db} / \mathrm{db}$ mice revealed significant differences in values of some of these biomarkers. For example, the levels of leptin, TIMP-1, RANTES, VEGF, MCP-5, MIP-1a, and TARC were 27, 3.2, 1.7, 1.6, 1.4, 1.3, and 1.2 times, respectively, higher in $\mathrm{db} / \mathrm{db}$ mice as compared to those in C57BL/6J mice. This test demonstrated a mixed effect for SBP consumption on the levels of these biomarkers in either animal models. For example, SBP-treated C57BL/6J mice had an approximately $35 \%$ reduction in the levels of sTNFRI, but no such effects of SBP were observed in $\mathrm{db} / \mathrm{db}$ mice. On the other hand, SBP treatment was associated with a significant reduction in the levels of IL-3 in either of animal models. While SBP treatment was associated with a significant reduction of approximately $15 \%$ in RANTES levels in $\mathrm{db} / \mathrm{db}$ mice, such effect was not observed in C57BL/6J mice.

\section{Discussion}

Epidemiological studies have indicated that diets rich in flavonoids from vegetables and fruits can reduce both incidence and progression of age related diseases. Saskatoon berries are known to have high levels of phytochemicals with antioxidant properties, including flavonoids. These berries are primarily produced in the Prairie Provinces of Canada, including Manitoba and Saskatchewan. Not only are they consumed as fruits, but also they are used in many other forms of food products such as pies, jams, muffins and others. In this study, we used Saskatoon Berry Powder (SBP) to investigate its efficacy in a mouse model of diabetes, namely $\mathrm{db} / \mathrm{db}$ mice as well as their wildtype counterparts $(\mathrm{C} 57 \mathrm{BL} / 6 \mathrm{~J})$. The $\mathrm{db} / \mathrm{db}$ mice lack leptin receptors 
Table 2: Plasma inflammatory-related biomarkers as estimated per fluorescent intensity in pooled plasma samples $(n=4)$ taken at the end of the study.

\begin{tabular}{|c|c|c|c|c|}
\hline \multirow[t]{2}{*}{ Cytokine Description } & \multicolumn{2}{|c|}{$\mathrm{db} / \mathrm{db}$ mice } & \multicolumn{2}{|c|}{ C57BL/6J mice } \\
\hline & Control & Treated & Control & Treated \\
\hline IL-3 & $631 \pm 41$ & $492 \pm 49^{a}$ & $529 \pm 60$ & $424 \pm 62^{a}$ \\
\hline $\mathrm{IL}-4$ & $922 \pm 88$ & $964 \pm 80$ & $849 \pm 54$ & $743 \pm 57$ \\
\hline $\mathrm{IL}-5$ & $370 \pm 24$ & $412 \pm 28$ & $331 \pm 72$ & $303 \pm 43$ \\
\hline IL-13 & $384 \pm 55$ & $359 \pm 12$ & $298 \pm 36$ & $221 \pm 12$ \\
\hline Leptin & $6,388 \pm 916^{b}$ & $6,437 \pm 620^{\mathrm{b}}$ & $233 \pm 82$ & $184 \pm 3$ \\
\hline MCP-5 & $1,221 \pm 102$ & $1,358 \pm 101$ & $914 \pm 397$ & $982 \pm 203$ \\
\hline MIP-1a & $275 \pm 11$ & $229 \pm 41$ & $206 \pm 32$ & $172 \pm 10$ \\
\hline MIP-2 & $975 \pm 90$ & $1,050 \pm 45$ & $848 \pm 195$ & $848 \pm 25$ \\
\hline RANTES & $202 \pm 12^{b}$ & $171 \pm 1^{\mathrm{a}, \mathrm{b}}$ & $120 \pm 15$ & $137 \pm 11$ \\
\hline $\mathrm{SCF}$ & $549 \pm 57$ & $430 \pm 75^{b}$ & $388 \pm 12$ & $244 \pm 32$ \\
\hline sTNFRI & $63,178 \pm 1,095$ & $64,047 \pm 1,332$ & $56,362 \pm 12,815$ & $37,555 \pm 7,671^{c}$ \\
\hline TARC & $948 \pm 130$ & $888 \pm 22^{b}$ & $757 \pm 28$ & $689 \pm 60$ \\
\hline TIMP-1 & $2,563 \pm 498^{b}$ & $2,854 \pm 494^{b}$ & $797 \pm 425$ & $629 \pm 185$ \\
\hline Thrombopoietin & $911 \pm 46$ & $969 \pm 44$ & $774 \pm 252$ & $767 \pm 87$ \\
\hline VEGF & $644 \pm 74^{b}$ & $650 \pm 52^{b}$ & $396 \pm 109$ & $332 \pm 38$ \\
\hline
\end{tabular}

IL: Interleukin; MCP: Monocyte Chemoattractant Protein; MIP: Macrophage Inflammatory Protein; RANTES: Regulated on Activation, Normal T Cell Expressed and Secreted; SCF: Stem Cell Factor; sTNFRI: Soluble Tumor Necrosis Factor Receptor I; TARC: Thymus- and Activation-Regulated Chemokine; TIMP-1: Tissue Inhibitor of Metalloproteinase; VEGF: Vascular Endothelial Growth Factor

$\mathrm{a}, \mathrm{db} / \mathrm{db}$-treated as compared to $\mathrm{db} / \mathrm{db}$ control

b, db/db as compared to corresponding C57BL/6J groups

c, C57BL/6J treated as compared to C57BL/6J control

Values are means $\pm S D ; n=4$

and thereby become obese and diabetic over time. Furthermore, they develop other abnormalities generally associated with obesity and diabetes such as dyslipidemia, imbalanced inflammatory pathways, and cardiovascular disorders. Due to severe obesity, these animals cannot be maintained for a long period of time.

An SBP dose of 5\% (w/w) was used based on our previous pilot experiment in which we observed this would be the best effective dose to reduce blood glucose levels in $\mathrm{db} / \mathrm{db}$ mice over 4 weeks [11]. Many clinical and experimental studies have reported that 4-week is sufficient to observe metabolic effects of dietary agents [10-13]. One factor that may indicate compliance with the experimental diets is food intake as well as normal growth and development of the animals. In these young animals, apparently normal body weight gain can be considered as a factor of normal growth and development. Thus, to provide evidence on acceptability of SBP we regularly monitored food intake and body weight gain in the mice. As it is shown in (Table 1), the $\mathrm{db} / \mathrm{db}$ mice had higher body weight at the baseline as compared to that in the wild-type mice; body weight gain over the 4 weeks of experiment was substantially more profound in $\mathrm{db} / \mathrm{db}$ mice than in wild-type mice. This substantial difference in body weight gain between the two animal models is most likely related to their food intakes. We observed a significantly higher food intake in $\mathrm{db} / \mathrm{db}$ mice as compared to that in their wild-type counterparts (C57BL/6J mice). Our data demonstrate that body weight was increased by $91 \%$ and $71 \%$ in $\mathrm{db} / \mathrm{db}$ mice and $19 \%$ and $16 \%$ in wild-type mice over the 4 -week of experimental course in the treated and control animals, respectively. These data suggest metabolic abnormalities in $\mathrm{db} / \mathrm{db}$ mice as well as potential efficacy of SBP in body weight management. We observed $20 \%$ and $16 \%$ reductions in body weight gain in $\mathrm{db} / \mathrm{db}$ and wild-type

mice that consumed SBP supplemented diet, respectively, by the end of the study. It also appears that the potential efficacy of SBP in body weight management could be at least in part through regulations in food intake, as both groups of mice treated with SBP had lower food intake as compared to their corresponding untreated controls.

We hereby report an approximately $30 \%$ reduction in whole blood glucose levels after 4 weeks of treatment with SBP in $\mathrm{db} / \mathrm{db}$ mice, but SBP had no such effects in wild-type mice. This effect could be related either to lower body weight or a direct effect of phytochemicals of SBP or a combination of two. On the other hand, this dietary supplementation did not significantly change plasma lipid levels. It should be mentioned that higher urine blood glucose levels in $\mathrm{db} / \mathrm{db}$ mice suggests a regulatory body mechanism on glucose metabolism that could result in higher excretion of urinary blood glucose in an attempt to maintain normal blood glucose levels. This observation may suggest the importance of monitoring urine blood glucose levels in subjects at risk for development of type-2 diabetes.

The state of obesity and type- 2 diabetes is considered to be commonly associated with imbalance in immune system and inflammatory markers. Our data support this notion as the $\mathrm{db} / \mathrm{db}$ mice showed significant differences in the parameters pertaining to inflammatory status as compared to those in their wild-type counterparts free of hyperglycemia and obesity. Furthermore, treatment with SBP was associated with alterations in these inflammatory markers mainly in $\mathrm{db} / \mathrm{db}$ mice. This may suggest a direct effect of effective phytochemicals present in SBP and/or an indirect effect because of alleviations in the status of obesity and diabetes observed by the end of the study. We have used an available 
inflammatory marker kit for experimental mice which tests samples for 21 inflammatory markers. It should be mentioned that not all of these 21 inflammatory markers are concerned to diabetes. However, we report that these markers show the potential effects of SBP on inflammatory systems in 2 different commonly-used mouse models.

\section{Conclusion}

This short-term study demonstrates very interesting evidence on potential effectiveness of SBP against type- 2 diabetes in $\mathrm{db} / \mathrm{db}$ mice. Approximately $30 \%$ reductions in plasma and urine glucose levels observed in the SBP-treated $\mathrm{db} / \mathrm{db}$ mice is very encouraging and warrants further investigations on potential mechanisms of action as well as longer treatment protocols in this and other animal models of type- 2 diabetes. It is not clear which component(s) of SBP is responsible for the effects observed in this study. Future studies should be carried out to identify such functional components of SBP.

\section{Acknowledgement}

The authors would like to thank St. Boniface Hospital Foundation for providing infrastructure, facilitating performance of these studies. This work was supported by research fund from Agricultural and Food Development Initiative- Growing Forward 2, Diabetes Canada and Natural Sciences and Engineering Research Council of Canada (NSERC).

\section{References}

1. Li J, Deng R, Hua X, Zhang L, Lu F, Coursey TG, et al. Blueberry component pterostilbene protects corneal epithelial cells from inflammation via antioxidative pathway. Sci Rep. 2016; 6: 19408.

2. Nardi GM, Januario AGF, Freire CG, Megiolaro F, Schneider K, Perazzoli MRA, et al. Anti-inflammatory activity of berry fruits in mice model of inflammation is based on oxidative stress modulation. Pharmacognosy Res. 2016; 8: S42-S49.

3. Huang W, Zhu Y, Li C, Sui Z, Min W. Effect of blueberry anthocyanins malvidin and glycosides on the antioxidant properties in endothelial cells. Oxid Med Cell Longev. 2016.
4. Pervin M, Hasnat MA, Lim JH, Lee YM, Kim EO, Um BH, et al. Preventive and therapeutic effects of blueberry (vaccinium corymbosum) extract against DSS-induced ulcerative colitis by regulation of antioxidant and inflammatory mediators. J Nutr Biochem. 2016; 28: 103-113.

5. Li Q, Wang J, Shahidi F. Chemical characteristics of cold-pressed blackberry, black raspberry, and blueberry seed oils and the role of the minor components in their oxidative stability. J Agric Food Chem. 2016; 64: 5410-5416.

6. Bingül I, Başaran-Küçükgergin $C$, Aydın AF, Soluk-Tekkeşin M, Olgaç $V$, Doğru-Abbasoğlu $S$, et al. Blueberry treatment attenuated cirrhotic and preneoplastic lesions and oxidative stress in the liver of diethylnitrosaminetreated rats. Intl J Immunopathol Pharmacol. 2016; 29: 426-437.

7. Pinto MS, de Carvalho JE, Lajolo FM, Genovese MI, Shetty K. Evaluation of antiproliferative, anti-type 2 diabetes, and antihypertension potentials of ellagitannins from strawberries (Fragaria $\times$ ananassa Duch.) using in vitro models. J Med Food. 2010; 13: 1027-1035.

8. Seeram NP, Adams LS, Zhang Y, Lee R, Sand D, Scheuller HS, et al. Blackberry, black raspberry, blueberry, cranberry, red raspberry, and strawberry extracts inhibit growth and stimulate apoptosis of human cancer cells in vitro. J Agric Food Chem. 2006; 54: 9329-9339.

9. Hosseinian FS, Beta T. Saskatoon and wild blueberries have higher anthocyanin contents than other Manitoba berries. J Agric Food Chem. 2007; 55: $10832-108388$.

10. Moghadasian $\mathrm{MH}$. Dietary phytosterols reduce cyclosporine induced hypercholesterolemia in apo E-KO mice. Transplantation. 2006; 81: 207-213.

11. Moghadasian MH, Frohlich JJ. Effects of dietary phytosterols on cholesterol metabolism and atherosclerosis: Clinical and experimental evidence. Am J Med. 1999; 107: 588-594.

12. Moghadasian MH, McManus BM, Pritchard PH, Frohlich JJ. "Tall oil"-derived phytosterols reduce atherosclerosis in apo E-deficient mice. Arterioscler Thromb Vasc Biol. 1997; 17: 119-126.

13. Zhao R, Le K, Li W, Ren S, Moghadasian MH, Beta T, et al. Effects of Saskatoon berry powder on monocyte adhesion to vascular wall of leptin receptor-deficient diabetic mice. J Nutr Biochem. 2014; 25: 851-857.
Austin J Nutri Food Sci - Volume 7 Issue 1 - 2019

ISSN : 2381-8980 | www.austinpublishing group.com Moghadasian et al. (C) All rights are reserved
Citation: Moghadasian MH, Masisi K, Le K, Beta T, Shen GX and Fischer G. The Potential Anti-Diabetic Effects of Saskatoon Berry in Experimental Mouse Models. Austin J Nutri Food Sci. 2019; 7(1): 1111. 